NEWS maker

\title{
Proteostasis Therapeutics
}

\author{
Why protein homeostasis has investors excited.
}

A shared interest in the biology of aging brought a chemist, a geneticist and a biologist together to found Proteostasis Therapeutics. In 2006, chemist Jeffery Kelly from The Scripps Research Institute began talking with worm geneticist Andrew Dillin, based at the Salk Institute, about exploiting new links between protein misfolding and diseases of aging. Proteostasis was launched, and soon after, molecular and cell biologist Richard Morimoto from Northwestern University in Evanston, Illinois, was brought in to complete the company's scientific foundations.

The Cambridge, Massachusetts-based startup takes its name from the concept that to ensure health, every cell and tissue must preserve protein homeostasis, or 'proteostasis'. Because proteostasis is preserved by a complex network of interacting biological processes, when the network is compromised, disease can result.

The company is pursuing 'proteostasis regulators' - single small molecules to nudge the cell's proteostasis network back to balance. The company concept was developed at an extra-innings San Diego Padres baseball game by Kelly, Dillin and Chris Mirabelli, managing director of HealthCareVentures in Cambridge, Massachusetts.

Intrigued by the idea, Mirabelli invited the researchers to Boston to make a presentation to his partners. HealthCare Ventures seeded the company for about 18 months, during which time Kelly and Dillin brought in Morimoto. Then in August 2008, a series A financing round followed, which closed on $\$ 45$ million.

Individual proteostasis regulators could, in principle, tackle multiple diseases involving protein misfolding or aggregation, from those caused by rare enzyme defects like Gaucher's and Fabry's to neurodegenerative diseases such as Alzheimer's, Huntington's and Parkinson's. The cell's ability to adjust proteostasis also wanes with age, a decline that the founders posit may partially explain degenerative diseases of aging.

The underlying premise-based on work in animal models-is that a single mutant protein can trigger, through progressive disruption, the collapse of protein homeostasis in the cell. For example, the polyglutamine repeat in Huntington's disease can tip an aging cell, struggling to maintain homeostasis, into a cascade of misfolding.

The company is pursuing small-molecule compounds that can potentially work on many of the 'nodes' in the network: the heat shock response, which guides the folding, trafficking, assembly and disassembly of proteins; the unfolded protein response, which stabilizes proteins in the endoplasmic reticulum (ER) before secretion; and compounds that increase ER calcium levels, which also enhance protein folding.

Proteostasis is Dillin and Morimoto's first company. Dillin, as a postdoc in Cynthia Kenyon's laboratory at the University of California, San Francisco, demonstrated in 2002 the role of mitochondrial function in aging and longevity. Morimoto cloned heat shock protein 70 (HSP70), in 1985, and has been a leader in the heat shock and chaperone field for a quarter century. He tried unsuccessfully to launch a heat shock protein company in the early 1990s but may have been too far ahead of the wave: little was known then about protein misfolding diseases and the connection to chaperones and stress responses.

Proteostasis is Kelly's second company. His first, FoldRx Therapeutics, appears headed for success after an impressive pivotal trial for its first drug, tafamadis meglumine (FX-1006A), in transthyretin (TTR) amyloid polyneuropathy, a fatal neurodegenerative disease (Nat. Biotechnol. $27,874,2009)$. In contrast to FoldRx's very specific chemical approach to disease, Proteostasis takes a global, biological approach. Its drugs don't target single-disease proteins but instead enhance a cell's network of proteins, an unlikely but logical approach for chemist Kelly, for whom biology has become an essential second vocation.

There's hardly scientific consensus that protein misfolding and aggregation themselves cause neurodegenerative diseases like Huntington's, says Henry Paulson, a neurologist and researcher at the University of Michigan in Ann Arbor. More subtle protein-specific changes might be responsible. But Paulson says there's compelling evidence from the Proteostasis founders and from others that abnormal protein conformations are a key to these diseases, and he favors the company's therapeutic approach of enhancing the cell's quality control machinery.

But how druggable is the cell's entire protein network? Morimoto and Kelly disclose that a screen for heat shock response activators has yielded compounds that are effective in animal disease models. Discovering useful compounds will not pose a problem, Kelly says. The hard

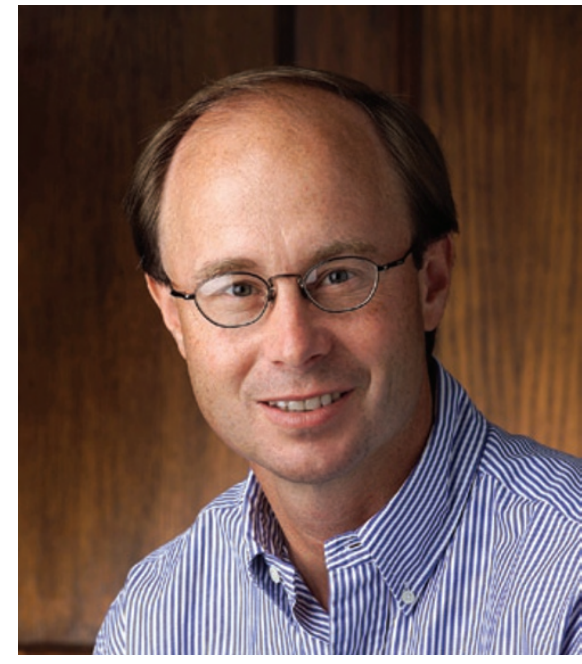

Jeffery Kelly, Proteostasis Therapeutics cofounder

part will be to pick the ideal disease in which to perform a clinical trial. The disease must be detected early enough to show the impact of an intervention, so in neurodegeneration the company may start with Huntington's, a disease which, in theory, can be identified at an early stage. Another focus is lysosomal storage diseases.

One challenge is side effects. Although enhancing the heat shock response and the unfolded protein response should be a good thing, these processes are also implicated in cancer. The company's goal is only to restore normal homeostasis, and Morimoto speculates that a $15-20 \%$ boost in chaperones should be effective without increasing cancer risk. Nonsteroidal anti-inflammatory drugs, which are potent activators of the heat shock transcription factor, do not cause cancer, he adds - a good sign. Another theoretical risk is viral infection, because enhancing a cell's protein production and secretion ability could aid viral replication.

On the benefit side, there's a remote but real chance that proteostasis regulators could slow the aging process, because genes involved in folding and misfolding also appear in pathways linked to life span. For example, Kelly and Dillin have reported that a decrease in insulin growth factor signaling has a profoundly beneficial effect on protein aggregation and toxicity in a worm model of Alzheimer's disease. Reduced insulin growth factor signaling extends life span in mice and other organisms. Could proteostasis regulators do this in humans? The company is not trying to slow the aging process - that may be too big a charge - but Morimoto stresses that its compounds are designed to restore the molecular processes that decline during aging.

Ken Garber Ann Arbor, Michigan 